\title{
A Thermopneumatic Micropump Based on Micro-engineering Techniques
}

\author{
F C M VAN DE POL, $\mathrm{H}$ T G VAN LINTEL, $M$ ELWENSPOEK and $J$ H $J$ FLUITMAN \\ Unwersuty of Twente, Department of Electrical Engineering, PO Box 217, 7500 AE Enschede (The Netherlands)
}

\begin{abstract}
The design, working principle and realization of an electro-thermopneumatic liquid pump based on micro-engineering techniques are described The pump, which is of the reciprocating displacement type, comprises a pump chamber, a thin silicon pump membrane and two silicon check valves to direct the flow The dynamic pressure of an amount of gas contained in a cavity, controlled by resistive heatıng, actuates the pump membrane The cavity, chambers, channels and valves are realized in silicon wafers by wet chemical etching Experımental results are presented Maximum yield and bult-up pressure equal $34 \mu \mathrm{l} / \mathrm{min}$ and $005 \mathrm{~atm}$, at a supply voltage of $6 \mathrm{~V}$ Results of simulations show good agreement with the actual dynamic behaviour of the pump
\end{abstract}

\section{Introduction}

Since 1983, research on microminiature pumps has been carned out at the University of Twente in the research group 'Transducers and Materials Science', which is part of the research unit 'Sensors \& Actuators' Startıng from the principle of a peristaltic piezoelectric pump, developed in 1980 at Stanford University by Wallmark and Smits [1], several prototypes have been realized, applying micromachining of silicon and using bimorph or monomorph piezoelectric discs for the actuation [2]

A major drawback of these piezoelectrically driven pumps is the required high electrical supply voltage (about $100 \mathrm{~V}$ ) Besides this, application of the piezoelectric discs interferes with integrated fabrication Aiming at the realization of a pump merely applyıng micro-engineering techniques like thin-film technology, photolithographic technıques and silıcon micromachining and considering various actuation principles, we concluded that thermopneumatic actuation was a good alternative This actuation principle has been used by Zdelblick and Angell in a microminiature valve, using a gas/liquid system and resistıve heatıng [3]
We realized and reported on a thermopneumatic actuator [4], comprising a cavity filled with air, a bult-in heater resistor and a flexible membrane Here, the mounting of such a thermopneumatic actuator on a pump body is discussed, and results for a complete, thermopneumatically driven, micropump are presented

\section{Design and Working Principle}

\section{Geometrical Destgn}

Figure 1 shows cross sections of the pump, comprising a pump chamber and two circular, silicon check valves The actuating part of the pump comprises a cavity filled with air, a square silicon pump membrane and a bult-in aluminium meander which serves as a heater resistor The resistor is supported by a thin silicon sheet suspended by four small silicon beams, which serve as thermal insulators Alumınium current leads connectıng the meander to the bond pads run through narrow channels, which form a restriction to gas flow The flexible membrane can displace liquid present in the pump chamber

Valves

Each valve consists of a circular, silicon diaphragm comprising a flexural outer ring and a

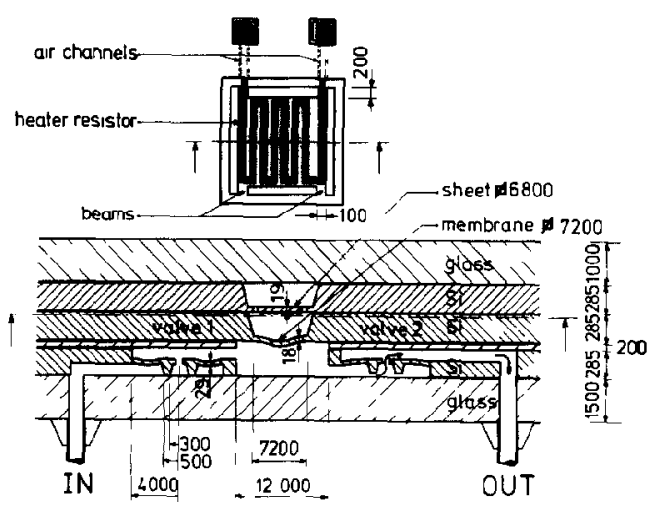

Fig 1 Cross-sectional views of the pump (dimensions in $\mu \mathrm{m}$ ) 
rigid inner sealing ning Because of the flexibility of the outer nng, a pressure actıng on the valve deflects the sealing ring $A$ thin oxide film on the summit of the sealing ning slightly deflects the diaphragm when the valve is closed, giving the valve a small pre-tension

\section{Working Principle}

The application of an electric voltage to the heater resistor causes a temperature nise of the air inside the cavity and a related pressure increase inducing a downward deflection of the pump membrane As the pump membrane deflects downwards, the pressure in the pump chamber is increased Provided this pressure exceeds the sum of the outlet pressure and the pre-tension of valve 2 , this valve will open Because of the pressure difference across the open valve 2, hquid flows through the gap underneath the sealing ring, thereby reducing the pressure in the pump chamber, while valve 1 checks the return flow to the inlet When the pump chamber pressure equals the sum of the pre-tension of valve 2 and the outlet pressure, valve 2 closes In the meantime, the pressure of the air inside the cavity, being higher than that outside, induces a flow of gas out of the cavity through the narrow air channels, causing this air pressure to decrease

When the voltage is switched off, the air inside the cavity cools down, causing a pressure drop in the cavity and a related upward deflection of the pump membrane As the pump membrane moves upwards, the pressure in the pump chamber decreases Provided the inlet pressure exceeds the sum of the pressure in the pump chamber and the pre-tension of valve 1 , this valve will open Because of the pressure difference across the open valve 1 , liquid flows through the gap underneath the sealing ring, thereby increasing the pressure in the pump chamber, while valve 2 checks the return flow from the outlet When the pressure in the pump chamber equals the inlet pressure minus the pretension of valve 1 , this valve closes In the meantıme, the pressure of the air inside the cavity, being lower than that outside, induces a flow of gas through the narrow air channels into the cavity, causing this air pressure to increase

\section{'Thermal' Response}

'Thermal' response (warming up and cooling down of the air inside the cavity) can be described by a 'thermal' relaxation time, $\tau_{t}$, which is determined by the thermal resistance of the air inside the cavity, the thermal resistance of the small suspending beams, and the total heat capacity of the cavity Since the heat capacity of the air is negligible compared to that of the suspended thin slicon sheet supporting the resistor, the total heat capacity approximately equals the heat capacity of the sheet

The maximum achievable temperature, $T_{\max }$, inside the cavity is determined by the input power and the thermal resistances mentioned For the pump described here, $\tau_{\mathrm{t}}$ equals $01 \mathrm{~s}$

\section{'Pneumatic' Relaxatton}

'Pneumatic' relaxation (increase or decrease of the air pressure in the cavity due to a flow of gas into or out of it, induced by a pressure difference over the alr channels) can be described (in approximation, since the pneumatic system is nonlinear) by a 'pneumatic' relaxation time, $\tau_{p}$, which is determined by the volume of the cavity and the flow resistance of the narrow air channels

The maximum achievable pressure inside the cavity, $p_{\max }$, is determined by $T_{\max }$ and $\tau_{\mathrm{p}}$, and decreases with $\tau_{p}$ However, if $\tau_{p} \gg \tau_{t}$, then $T_{\max }$ is almost independent of $\tau_{p}$ For the pump described here, $\tau_{p}$ equals $5 s\left(\gg \tau_{t}\right)$

\section{'Hydraulic' Relaxatzon}

'Hydraulic' relaxation (increase or decrease of the pump chamber pressure due to a flow of liquid into or out of the pump chamber, induced by pressure differences over the valves) is determined by the volume stroke of the pump membrane and the flow resistances of the valves Since the valves show highly nonlınear behaviour, this relaxation cannot be described by a single relaxation time Nevertheless, we can state that the hydraulic relaxation, for the pump described here, is (much) slower than the thermal response, and (much) faster than the pneumatic relaxation The pumped volume in one pump stroke, $\Delta V_{\mathrm{ps}}$, is determined by $p_{\max }$, the pump frequency, and the hydraulic relaxation If the hydraulic relaxation is much faster than the pneumatic relaxation, $\Delta V_{p s}$ is almost independent of $\tau_{p}$, and is determined by the speed of the hydraulic relaxation and the pump frequency

The maximum liquid volume, pumped during one pump stroke, $\Delta V_{\mathrm{ps,max}}$, equals the difference in pump chamber volumes at the end of a (hydraulically relaxed) downward stroke, and at the end of a (hydraulically relaxed) upward stroke of the pump membrane

\section{Simulation}

\section{Bond Graph Techniques and TUTSIM}

Bond graph techniques [5] are used for physical modelling of the pump The model comprnses the thermal behaviour of the pump, the thermo- 
dynamics of the air inside the cavity, the fluid dynamics of the air and hquid channels, and the mechanics of the flexible pump membrane and valves Simulation of the dynamic pump behaviour is carried out with TUTSIM, a simulation program developed at the University of Twente A detailed description of the bond graph model will be reported elsewhere

\section{Experiments}

\section{Realization}

Starting materials for the pump are three (100) 2-in silicon wafers, polished on both sides, and three Duran borosilicate glass wafers The slicon wafers are shaped by wet chemical etching in a KOH water solution using standard photolithographic techniques for pattern definition The glass wafers are cut out off a plate and polished (surface roughness $<005 \mu \mathrm{m}$ )

The silicon wafers are attached to one another by anodic bonding, using intermediate layers of silicon oxide and sputtered borosilicate glass [6] The silicon and glass wafers are attached by direct anodic bonding $[2,7]$ The oxide on the summit of the sealing rings of the valves not only provides them with a pre-tension, but also prevents them becoming bonded to the glass 'selective bonding' [2]

An evaporated aluminium film, patterned by wet chemical etching, is used for the heater resistor More details on manufacturing and technology can be found in refs 2 and 4 The dimensions of the pump are given in Fig 1

\section{Priming}

The pump is not self-priming It can be filled straightforwardly by injecting water in the inlet and/or extractung alr at the outlet, with the aid of a syringe However, if this priming method is applied, air bubbles often remain inside the pump chamber or channels, obstructing the proper functioning of the valves or affecting the pump behaviour The best priming procedure appears to be the one described in ref 2 the pump is submerged in water in a bell jar As the bell jar is evacuated, the air in the pump is removed Then, as air is admitted, the pump fills with water

\section{Measurements}

In Fig 2, the measurement set-up is depicted A pulsed $\mathrm{d} c$ voltage, vanable in height and frequency, is applied to the bonding pads connected to the heater resistor Pumped volume and yield are measured with the aid of a glass capillary tube and a stopwatch Specific pressures at the inlet

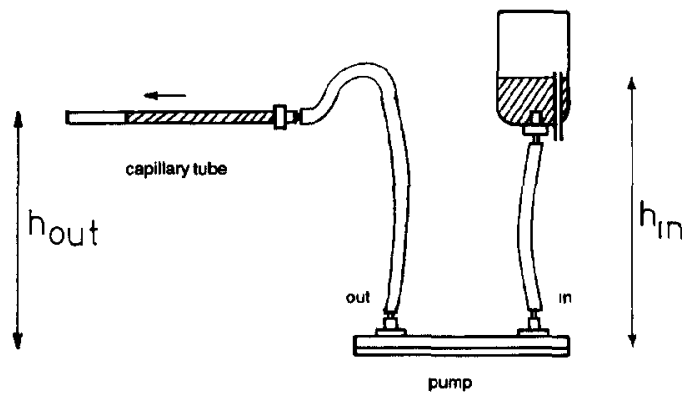

F1g 2 Measurement set-up

and outlet of the pump are established by adjusting $h_{\text {out }}$ and $h_{\mathrm{un}}$

The working of the valves is characterized by measurning spontaneous flow (no voltage applied) as a function of inlet and outlet pressures

Dust or precipitation of dissolved particles might interfere with the proper working of the valves or, in the most severe case, even cause stoppage of the channels or tubes Therefore, the pump, the water reservoir and the capillary tube are placed in a laminar flow box Deionized, filtered water is used The use of degassed (in an evacuated bell jar) water helps to prevent the formation of air bubbles inside the pump

\section{Results and Discussion}

Figure 3 shows the pump yield as a function of pump frequency, applied voltage and back pressure (outlet pressure minus inlet pressure), for a constant inlet pressure of $1 \mathrm{~atm}$ Measured results appeared to be reproducible within 5\%

For a given back pressure and supply voltage, see Fig 3(a), at first the yield mcreases almost linearly with pump frequency, reaches a maximum for $f_{m}$, and then decreases to zero In the linear part, yleld is determined by $p_{\max }\left(T_{\max }\right)$ and pump frequency $\left(\Delta V_{\mathrm{ps}} \simeq \Delta V_{\mathrm{ps} \max }\right)$ The frequency $f_{\mathrm{m}}$ is determined by the speed of hydraulic relaxation at higher frequencies, there is not enough time for hydraulic relaxation $\left(\Delta V_{\mathrm{ps}}<\Delta V_{\mathrm{ps} \text {,max }}\right)$ The frequency $f_{\mathrm{m}}$ increases with the supply voltage and decreases with the back pressure, because of the nonlinear flow resistances of the valves

In Fig 3(b), a number of pump curves are presented for vanous applied voltages and pump frequencies The dashed curves in Fig 3 represent the results of simulations, agreeing within some $15 \%$ with measured yields Simulated temperature nse and pressure rise inside the cavity equal $30^{\circ} \mathrm{C}$ and $006 \mathrm{~atm}$, for a dc supply voltage of $6 \mathrm{~V}$ Required electrical energy per pumped volume equals a few $J / \mu 1$, depending on pump frequency and back pressure 


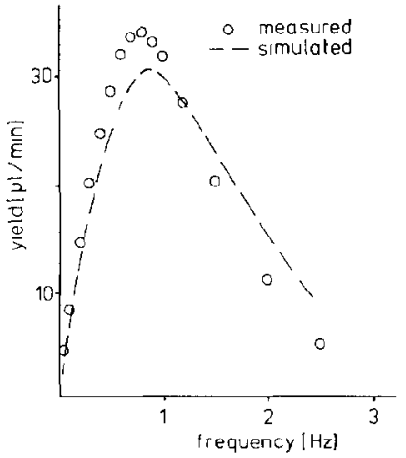

(a)

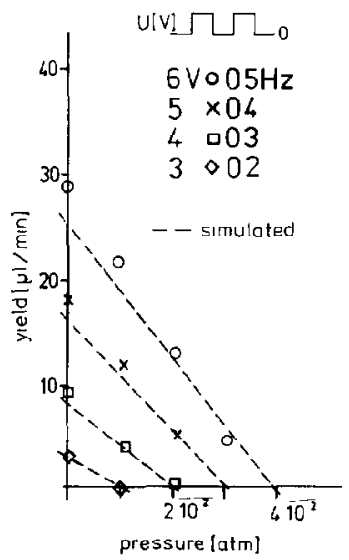

(b)

Fig 3 (a) Pump yield as a function of pump frequency for an applied voltage of $6 \mathrm{~V}$ at zero back pressure (outlet minus inlet pressure) (b) Pump yeld as a function of back pressure, applied voltage and pump frequency

Figure 4 shows measured and simulated spontaneous flow as a function of inlet pressure minus outlet pressure If outlet pressure exceeds inlet pressure minus the pre-tensions of the valves, no measurable flow occurs, mdicating that the

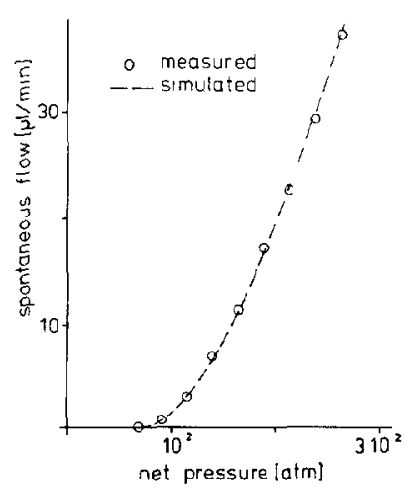

Fig 4 Measured and simulated spontaneous flow vs net pressure (inlet minus outlet pressure) closure of the valves is very good Calculated pre-tension of one valve equals $10^{-3} \mathrm{~atm}$

\section{Conclusions}

The design, working principle and realization of an electro-thermopneumatic micropump are described The pump compnses a pump chamber, a thin silicon pump membrane and two silicon check valves The pump membrane is actuated by the dynamic pressure of an amount of gas contained in a cavity, controlled by resistive heating Results of measurements and simulations of the dynamic behaviour of the pump are presented and discussed

The yield can be regulated by varying the pulsed d c supply voltage in height and/or frequency The pump behaves as expected simulated and measured yields, as functions of back pressure, supply voltage and pump frequency, agree withın some $15 \%$ Maximum yield and bult-up pressure equal $34 \mu \mathrm{l} / \mathrm{mIn}$ and $005 \mathrm{~atm}$, for a supply voltage of $6 \mathrm{~V}$

Important parameters are the thermal, pneumatic and hydraulic relaxation tımes, determining the dynamic behaviour of the pump Temperature rise and related pressure increase inside the cavity are a few tens of ${ }^{\circ} \mathrm{C}$ and some hundredths of an atmosphere The required electrical energy per pumped volume equals a few $J / \mu 1$, depending on pump frequency, back pressure and supply voltage Supply voltage and power consumption are less than $10 \mathrm{~V}$ and $2 \mathrm{~W}$ respectively

Dust or air bubbles readily interfere with the proper functioning of the valves and strongly affect the pump behaviour The closure of the valves is very good The construction of the pump is simple and it can be fabricated by merely applyıng micro-engineerıng techniques like thinfilm technology, photolithographic techniques and silicon micromachıning

\section{Acknowledgements}

The authors would like to thank Gert-Jan Burger, Rob Legtenberg, Dick Ekkelkamp, John Baxter and Peter Breedveld for their help and contributions

\section{References}

1 J G Smits, Piezoelectric micropump with three valves working peristaltically, Sensors and Actuators, A21-A23 (1990) 203-206

2 H T G van Lintel, F C M van de Pol and S Bouwstra, A piezoelectnc micropump based on micromachining of silicon, Sensors and Actuators, 15 (1988) 153-167 
$3 \mathrm{M} J$ Zdeblick and J B Angell, A microminiature electricto-fluidic valve, Proc 4ih Int Conf Solud-State Sensors and Actuators (Transducers '87), Tokyo, Japan, June 2-5, 1987, pp 827-829

4 F C $M$ van de Pol, D G J Wonnunk, M Elwenspoek and J H J Flutman, A thermo-pneumatic actuation principle for a microminiature pump and other micromechanical devices, Sensors and Actwators, 17 (1989) 139-143
5 A M Bos and P C Breedveld, 1985 update of the bond graph bibliography, $J$ Franklin Inst, 319 (1985) 269-286

6 A Hanneborg and P A Qhlckers, Anodic bonding of silicon chips using sputter-deposited Pyrex 7740 thin films, Proc 12th Nordic Semiconductor Meeting, Jeunaker, Norway, June 8-11, 1986, pp 290-293

$7 \mathrm{G}$ Wallis and D I Pomerantz, Field assisted glass-metal sealing J Appl Phys, 40 (1969) 3946-3949 